\title{
On the Fractional Nagumo Equation with Nonlinear Diffusion and Convection
}

\author{
Abdon Atangana ${ }^{1}$ and Suares Clovis Oukouomi Noutchie ${ }^{2}$ \\ ${ }^{1}$ Institute for Groundwater Studies, Faculty of Natural and Agricultural Sciences, University of the Free State, \\ Bloemfontein 9300, South Africa \\ ${ }^{2}$ Ma SIM Focus Area, North-West University, Mafikeng 2735, South Africa
}

Correspondence should be addressed to Abdon Atangana; abdonatangana@yahoo.fr

Received 2 July 2014; Accepted 20 July 2014; Published 5 August 2014

Academic Editor: Dumitru Baleanu

Copyright (C) 2014 A. Atangana and S. C. Oukouomi Noutchie. This is an open access article distributed under the Creative Commons Attribution License, which permits unrestricted use, distribution, and reproduction in any medium, provided the original work is properly cited.

\begin{abstract}
We presented the Nagumo equation using the concept of fractional calculus. With the help of two analytical techniques including the homotopy decomposition method (HDM) and the new development of variational iteration method (NDVIM), we derived an approximate solution. Both methods use a basic idea of integral transform and are very simple to be used.
\end{abstract}

\section{Introduction}

The Nagumo equation with linear or nonlinear diffusion and convection has broadly been useful to population dynamics, ecology, neurophysiology, chemical reactions, and flame propagation [1-3]. In particular, the case where the equation involves degenerate nonlinear diffusion is of considerable interest [4-8]. In this case, a travelling wave front solution of sharp type is known to exist for exactly one value of the wave speed. Such wave fronts, for instance, represent collective motion of populations in particular collective cell spreading, invasion in ecology, and concentration in chemical reactions [9-15]. However, it has been showing that the real world problems describe via fractional order derivative gives better prediction [16-20]. It is, therefore, important; further extend the nonlinear Nagumo equation using the concept of fractional derivative order.

It is something very difficult to obtain the exact solution of nonlinear equation with fractional order derivative. Many scholars sometimes, to avoid this difficulty, solve this class of problem numerically. However, even with numerical scheme it is also difficult to provide a numerical solution for nonlinear equations. Thus, many scholars, to access the behaviour of the solution of the real problem under study, present an approximate solution of this type of equations.
In the literature, there exist several analytical techniques [21-25] to deal with nonlinear equations including fractional type. The purpose of this work is to present an approximate solution for the generalized nonlinear Nagumo equation withnonlinear diffusion and convection via the well-known variational iteration method (VIM) and the homotopy decomposition method (HDM). The nonlinear fractional Nagumo equation considered here is given below as

$$
\begin{aligned}
& \frac{\partial^{\alpha} u}{\partial t^{\alpha}}+\beta u^{n} \frac{\partial u}{\partial x}=\frac{\partial}{\partial x}\left[\alpha u^{n} \frac{\partial u}{\partial x}\right] \\
&+\gamma u\left(1-u^{m}\right)\left(u^{m}-\delta\right), \quad 0<\alpha \leq 1, \\
& u(x, 0)=f(x), \\
& u(0, t)=g(t),
\end{aligned}
$$

where $\alpha, \beta, \gamma$, and $\delta$ are constants, and for the sake of simplicity in this paper, we consider $m=n=1=\delta$. The concept of fractional order is not well by some scholars; in order to accommodate those, we present in the next section the basic information regarding this concept. 


\section{Basic Information about Fractional Calculus}

There exists a vast literature on different definitions of fractional derivatives. The most popular ones are the RiemannLiouville and the Caputo derivatives. For Caputo we have the following.

Definition 1 (see [26-30]). A real function $f(x), x>0$, is said to be in the space $C_{\mu}, \mu \in \mathbb{R}$ if there exists a real number $p>\mu$, such that $f(x)=x^{p} h(x)$, where $h(x) \in C[0, \infty)$, and it is said to be in space $C_{\mu}^{m}$ if $f^{(m)} \in C_{\mu}, m \in \mathbb{N}$.

Definition 2 (partial derivatives of fractional order [31-34]). Assume now that $f(x)$ is a function of $n$ variables $x_{i}, i=$ $1, \ldots, n$ also of class $C$ on $D \in \mathbb{R}_{n}$. We define partial derivative of order $\alpha$ for $f$ with respect to $x_{i}$, the function

$$
a \partial_{\underline{x}}^{\alpha} f=\left.\frac{1}{\Gamma(m-\alpha)} \int_{a}^{x_{i}}\left(x_{i}-t\right)^{m-\alpha-1} \partial_{x_{i}}^{m} f\left(x_{j}\right)\right|_{x_{j}=t} d t,
$$

where $\partial_{x_{i}}^{m}$ is the usual partial derivative of integer order $m$.

Definition 3 (see [26-30]). The Riemann-Liouville fractional integral operator of order $\alpha \geq 0$, of a function $f \in C_{\mu}, \mu \geq-1$, is defined as

$$
\begin{gathered}
J^{\alpha} f(x)=\frac{1}{\Gamma(\alpha)} \int_{0}^{x}(x-t)^{\alpha-1} f(t) d t, \quad \alpha>0, x>0, \\
J^{0} f(x)=f(x) .
\end{gathered}
$$

Properties of the operator can be found in [31-38]; we mention only the following.

For

$$
\begin{array}{cl}
f \in C_{\mu}, \quad & \mu \geq-1, \quad \alpha, \beta \geq 0, \quad \gamma>-1, \\
& J^{\alpha} J^{\beta} f(x)=J^{\alpha+\beta} f(x), \\
& J^{\alpha} J^{\beta} f(x)=J^{\beta} J^{\alpha} f(x), \\
& J^{\alpha} x^{\gamma}=\frac{\Gamma(\gamma+1)}{\Gamma(\alpha+\gamma+1)} x^{\alpha+\gamma} .
\end{array}
$$

\section{Basic Information of the HDM and VIM}

In this section, we shall present the basic information of the chosen analytical methods: the homotopy decomposition method and variational iteration method; we shall start with the homotopy decomposition method.

3.1. Information about the HDM. The interested reader can find the full detail of the methodology of the homotopy decomposition method in [39-42]. This relatively new method was recently used to solve some nonlinear fractional partial differential equations. However, since the new development of the variational iteration method using the Laplace transform was recently introduced, we shall present its methodology in the following subsection.
3.2. Some Information about the Variational Iteration Method. In its initial development, the essential nature of the method was to construct the following correction functional for (2) when $\alpha$ is a natural number:

$$
\begin{aligned}
& w_{n+1}(x, t)=w_{n}(x, t) \\
&+\int_{0}^{t} \lambda(t, \tau)\left[-\frac{\partial^{m} w(x, \tau)}{\partial t^{m}}+L\left(w_{n}(x, \tau)\right)\right. \\
&\left.+N\left(w_{n}(x, \tau)\right)+k(x, \tau)\right] d \tau
\end{aligned}
$$

where $\lambda(t, \tau)$ is the so-called Lagrange multiplier [43] and $w_{n}(x, t)$ is the $n$-approximate solution. However, this development was not suitable for equations with fractional order derivative [43]. Therefore, in their work, they apply the new development of the VIM proposed in [44] to find the Lagrange multiplier. In this new VIM, the first step of the basic character of the method is to apply the Laplace transform on both sides of (2) to obtain

$$
\begin{aligned}
& s^{m} w(x, s)-s^{m-1} w(x, 0)-\cdots w^{m-1}(x, 0) \\
& =\mathscr{L}[L(w(x, t))+N(w(x, t))+k(x, t)] .
\end{aligned}
$$

The recursive formula of (8) can now be used to put forward the main recursive method connecting the Lagrange multiplier as

$$
\begin{aligned}
& w_{n+1}(x, s) \\
& =w_{n}(x, s) \\
& \quad+\lambda(s)\left[s^{m} w_{n}(x, s)-s^{m-1} w(x, 0)-\cdots w^{m-1}(x, 0)\right. \\
& \left.\quad-\mathscr{L}\left[L\left(w_{n}(x, t)\right)+N\left(w_{n}(x, t)\right)+k(x, t)\right]\right] .
\end{aligned}
$$

Now considering $\mathscr{L}\left[L\left(w_{n}(x, t)\right)+N\left(w_{n}(x, t)\right)+k(x, t)\right]$, the restricted term; the Lagrange multiplier can be obtained as [43]

$$
\lambda(s)=-\frac{1}{s^{m}} .
$$

Now, applying the inverse Laplace transform on both sides of (9), we obtain the following iteration:

$$
\begin{aligned}
& w_{n+1}(x, t) \\
& =w_{n}(x, t) \\
& -\mathscr{L}^{-1}\left[\frac { 1 } { s ^ { m } } \left[s^{m} w_{n}(x, s)-s^{m-1} w(x, 0)-\cdots w^{m-1}(x, 0)\right.\right. \\
& -\mathscr{L}\left[L\left(w_{n}(x, t)\right)\right. \\
& \left.\left.\left.+N\left(w_{n}(x, t)\right)+k(x, t)\right]\right]\right] .
\end{aligned}
$$




\section{Application to the Fractional Nagumo Equation}

In this section, we present the application of the homotopy decomposition method and the new development of the socalled variational iteration method to the nonlinear fractional Nagumo equation. We shall start with the HDM.

4.1. Application of the HDM. Let us consider the fractional nonlinear Nagumo Equation with the following initial condition:

$$
\begin{gathered}
u(x, 0)=x^{2}, \\
\frac{\partial^{\alpha} u}{\partial t^{\alpha}}+\beta u \frac{\partial u}{\partial x}=\frac{\partial}{\partial x}\left[a u \frac{\partial u}{\partial x}\right]+\gamma u(1-u)(u-1), \\
0<\alpha \leq 1 .
\end{gathered}
$$

Using the steps involved in the HDM we arrive at the following:

$$
\begin{aligned}
& u(x, t) \\
& =u(x, 0) \\
& +\frac{1}{\Gamma[1-\alpha]} \int_{0}^{t}(t-\tau)^{1-\alpha}\left[-\beta u \frac{\partial u}{\partial x}+\frac{\partial}{\partial x}\left[a u \frac{\partial u}{\partial x}\right]\right. \\
& +\gamma u(1-u)(u-1)] d \tau .
\end{aligned}
$$

Now, assume the solution of the above equation can be expressed in series form as follows:

$$
u(x, t)=\sum_{n=0}^{\infty} p^{n} u_{n}(x, t) .
$$

Replacing this in (13) and after comparing the term of the same power of $p$, we obtain the following recursive formulas:

$$
u_{0}(x, t)=u(x, t)
$$

$$
\begin{array}{r}
u_{1}(x, t) \\
=\frac{1}{\Gamma[\alpha]} \int_{0}^{t}(t-\tau)^{\alpha-1}\left[-\beta u_{0} \frac{\partial u_{0}}{\partial x}+\frac{\partial}{\partial x}\left[a u_{0} \frac{\partial u_{0}}{\partial x}\right]\right. \\
\left.+\gamma u_{0}\left(1-u_{0}\right)\left(u_{0}-1\right)\right] d \tau
\end{array}
$$

Here,

$$
\begin{aligned}
& H_{2}^{1}=u_{0}(x, t) \partial_{x} u_{1}(x, t)+u_{1}(x, t) \partial_{x} u_{0}(x, t), \\
& H_{2}^{2}=2 \partial_{x} u_{0}(x, t) \partial_{x} u_{1}(x, t), \\
& H_{2}^{3}=u_{0}(x, t) \partial_{x x} u_{1}(x, t)+u_{1}(x, t) \partial_{x x} u_{0}(x, t),
\end{aligned}
$$

$$
\begin{aligned}
& H_{2}^{4}=u_{0}^{2}(x, t) u_{1}(x, t)+u_{1}^{3}+u_{1}^{2}(x, t) u_{0}(x, t), \\
& H_{2}^{5}=2 u_{0}(x, t) u_{1}(x, t) .
\end{aligned}
$$

The general recursive formula for $p \geq 3$ is given as

$$
\begin{aligned}
& u_{n}(x, t) \\
& =\frac{1}{\Gamma[\alpha]} \int_{0}^{t}(t-\tau)^{\alpha-1}\left[-\beta H_{n}^{1}+a H_{n}^{2}+a H_{n}^{3}\right. \\
& \left.+\gamma H_{n}^{4}+\gamma H_{n}^{5}-u_{n-1}\right] d \tau
\end{aligned}
$$

with

$$
\begin{aligned}
& H_{n}^{1}(x, t)=\sum_{j=0}^{n-1} u_{j}(x, t) \partial_{x} u_{n-j}(x, t), \\
& H_{n}^{2}(x, t)=\sum_{j=0}^{n-1} \partial_{x} u_{j}(x, t) \partial_{x} u_{n-j}(x, t), \\
& H_{n}^{3}(x, t)=\sum_{j=0}^{n-1} u_{j}(x, t) \partial_{x x} u_{n-j}(x, t), \\
& H_{n}^{4}(x, t)=\sum_{j=0}^{n-1} \sum_{k=0}^{j} u_{j}(x, t) u_{j-k}(x, t) u_{n-j-1}(x, t), \\
& H_{n}^{5}(x, t)=\sum_{j=0}^{n-1} u_{j}(x, t) u_{n-j}(x, t),
\end{aligned}
$$

so that, integrating the above set of integral equations, we obtain the following:

$$
\begin{aligned}
& u_{0}(x, t)=x^{2}, \\
& u_{1}(x, t)=-\frac{t^{\alpha} x^{2}\left(-6 a+2 x \beta+\left(-1+x^{2}\right)^{2} \gamma\right)}{\Gamma(1+\alpha)}, \\
& u_{2}(x, t) \\
& =t^{2 \alpha} x^{2}\left(\left(48 a^{2}+\gamma\right.\right. \\
& -2 a\left(3+24 x \beta+4 \gamma-22 x^{2} \gamma\right. \\
& +x\left(10 x \beta^{2}\right. \\
& +x \gamma\left(-2+x^{2}-2 \gamma+3 x^{2} \gamma-x^{6} \gamma\right) \\
& \left.\left.\left.+2 \beta\left(1+\left(2-8 x^{2}+3 x^{4}\right) \gamma\right)\right)\right)\right) \\
& \times \frac{1}{\Gamma(1+2 \alpha)}
\end{aligned}
$$




$$
\begin{aligned}
& +\frac{t^{\alpha} x^{4} \gamma\left(-6 a+2 x \beta+\left(-1+x^{2}\right)^{2} \gamma\right)^{2} \Gamma(1+2 \alpha)}{\Gamma^{2}(1+\alpha) \Gamma(1+3 \alpha)} \\
& \left.-\frac{t^{2 \alpha}\left(-6 a+2 x \beta+\left(-1+x^{2}\right)^{2} \gamma\right)^{3} \Gamma(1+3 \alpha)}{\Gamma^{3}(1+\alpha) \Gamma(1+4 \alpha)}\right) .
\end{aligned}
$$

Here, we have computed only three terms in the series solution. However, using the recursive formula, we can compute the remaining terms, and the approximate solution is given as follows:

$$
u(x, t)=u_{0}(x, t)+u_{1}(x, t)+u_{2}(x, t)+\cdots+\cdots .
$$

4.2. New Development of the Variational Iteration Method. In this subsection, we test the efficiency of the new development of the variation iteration method by solving the nonlinear fractional equation (1). Therefore, following the methodology of NDVIM, we are at the following.

The Lagrange multiplier is

$$
\lambda(s)=-\frac{1}{s^{\alpha}}
$$

and the recursive formula is given as

$$
\begin{aligned}
& u_{n+1}(x, t) \\
& =u_{n}(x, t) \\
& -\mathscr{L}^{-1}\left[\frac { 1 } { s ^ { \alpha } } \left[\mathscr { L } \left[-\beta u_{n} \frac{\partial u_{n}}{\partial x}\right.\right.\right. \\
& +\frac{\partial}{\partial x}\left[a u_{n} \frac{\partial u_{n}}{\partial x}\right] \\
& \left.\left.\left.+\gamma u_{n}\left(1-u_{n}\right)\left(u_{n}-1\right)\right]\right]\right]
\end{aligned}
$$

with the initial term

$$
u_{0}(x, t)=x^{2},
$$

so that using the iteration formulas we obtain

$$
\begin{aligned}
& u_{1}(x, t)=x^{2}-\frac{t^{\alpha}}{\Gamma(1+\alpha)} \\
& \times\left(6 a x^{2}-2 x^{3} \beta+x^{2}\left(1-x^{2}\right)\left(x^{2}-1\right) \gamma\right), \\
& u_{2}(x, t) \\
& =\left(x ^ { 2 } \left(t ^ { 3 \alpha } \left(-144 a^{3}\right.\right.\right. \\
& +12 a^{2} \\
& \times\left(18 x \beta+4 \gamma-22 x^{2} \gamma+21 x^{4} \gamma\right) \\
& -4 a\left(12 x \beta \gamma-54 x^{3} \beta \gamma\right. \\
& +48 x^{5} \beta \gamma+\gamma^{2} \\
& +43 x^{4} \gamma^{2}-48 x^{6} \gamma^{2}+18 x^{8} \gamma^{2} \\
& \left.+2 x^{2}\left(12 \beta^{2}-7 \gamma^{2}\right)\right)
\end{aligned}
$$

$$
\begin{aligned}
& +x\left(2 \beta \gamma^{2}+52 x^{4} \beta \gamma^{2}-52 x^{6} \beta \gamma^{2}\right. \\
& +18 x^{8} \beta \gamma^{2}+26 x^{7} \gamma^{3}-14 x^{9} \gamma^{3} \\
& +3 x^{11} \gamma^{3}+4 x^{2}\left(3 \beta^{3}-5 \beta \gamma^{2}\right) \\
& +6 x^{5}\left(5 \beta^{2} \gamma-4 \gamma^{3}\right)+2 x\left(5 \beta^{2} \gamma-\gamma^{3}\right) \\
& \left.\left.+x^{3}\left(-36 \beta^{2} \gamma+11 \gamma^{3}\right)\right)\right) \\
& \times \Gamma(1+\alpha) \Gamma^{2}(1+2 \alpha) \Gamma(1+4 \alpha) \\
& +t^{2 \alpha}\left(180 a^{2}+6 x \beta \gamma-20 x^{3} \beta \gamma+14 x^{5} \beta \gamma\right. \\
& +\gamma^{2}+12 x^{4} \gamma^{2}-10 x^{6} \gamma^{2}+3 x^{8} \gamma^{2} \\
& -4 a(23 x \beta+9 \gamma \\
& \left.-25 x^{2} \gamma+16 x^{4} \gamma\right) \\
& \left.+2 x^{2}\left(5 \beta^{2}-3 \gamma^{2}\right)\right) \\
& \times \Gamma(1+3 \alpha) \Gamma^{3}(1+\alpha) \Gamma(1+4 \alpha) \\
& +\Gamma(1+2 \alpha) \Gamma(1+3 \alpha) \\
& \times\left(t^{4 \alpha} x^{4} \gamma\right. \\
& \times\left(-6 a+2 x \beta+\gamma-2 x^{2} \gamma+x^{4} \gamma\right)^{3} \\
& \times \Gamma(1+3 \alpha) \\
& -\left(2 t^{\alpha}\left(16 a-2 x \beta-\gamma+2 x^{2} \gamma-x^{4} \gamma\right)\right. \\
& -\Gamma(1+\alpha)) \Gamma(1+\alpha) \\
& \left.\left.\left.\times \Gamma^{3}(1+\alpha) \Gamma(1+4 \alpha)\right)\right)\right) \\
& \times\left(\Gamma^{3}(1+\alpha) \Gamma(1+2 \alpha)\right. \\
& \times \Gamma(1+3 \alpha) \Gamma(1+4 \alpha))^{-1} .
\end{aligned}
$$

Using the recursive formula, the remaining term can be obtained but here, due to the length of this term, we computed only three terms and the approximate solution case given as

$$
u(x, t)=u_{2}(x, t)
$$

In the following section, we compare the approximate solution via HDM and NDVIM.

\section{Numerical Results}

We devote this section to the comparison of the numerical solutions obtained via the HDM and the NDVIM for different values of the fractional order derivative. In this case, we chose $\alpha=1.5, \gamma=1$, and $a=4$. The following figures show the numerical solution of the time fractional nonlinear 
TABLE 1: Comparison of numerical values for the approximate solutions via HDM and NDVIM.

\begin{tabular}{|c|c|c|c|c|c|}
\hline$x$ & $t$ & $\begin{array}{c}\text { HDM } \\
\alpha=0.25\end{array}$ & $\begin{array}{l}\text { NDVIM } \\
\alpha=0.25\end{array}$ & $\begin{array}{c}\text { HDM } \\
\alpha=0.9\end{array}$ & $\begin{array}{c}\text { NDVIM } \\
\alpha=0.9\end{array}$ \\
\hline \multirow{5}{*}{-10} & 0 & 100 & 100 & 100 & 100 \\
\hline & 1 & $-1.14275 \times 10^{18}$ & $-1.14319 \times 10^{18}$ & $-3.24381 \times 10^{17}$ & $-3.24545 \times 10^{17}$ \\
\hline & 2 & $-2.28554 \times 10^{18}$ & $-2.28628 \times 10^{18}$ & $-3.93359 \times 10^{18}$ & $-3.93465 \times 10^{18}$ \\
\hline & 6 & $-6.85676 \times 10^{18}$ & $-6.85845 \times 10^{18}$ & $-2.05326 \times 10^{20}$ & $-2.05347 \times 10^{20}$ \\
\hline & 10 & $-1.1428 \times 10^{19}$ & $-1.14305 \times 10^{19}$ & $-1.29153 \times 10^{21}$ & $-1.29161 \times 10^{21}$ \\
\hline \multirow{5}{*}{-5} & 0 & 25 & 25 & 25 & 25 \\
\hline & 1 & $-2.98096 \times 10^{12}$ & $-2.99989 \times 10^{12}$ & $-8.45746 \times 10^{11}$ & $-8.52756 \times 10^{11}$ \\
\hline & 2 & $-5.9636 \times 10^{12}$ & $-5.99543 \times 10^{12}$ & $-1.02663 \times 10^{13}$ & $-1.03118 \times 10^{13}$ \\
\hline & 6 & $-1.78972 \times 10^{13}$ & $-1.79697 \times 10^{13}$ & $-5.36272 \times 10^{14}$ & $-5.37156 \times 10^{14}$ \\
\hline & 10 & $-2.98327 \times 10^{13}$ & $-2.99391 \times 10^{13}$ & $-3.37376 \times 10^{15}$ & $-3.37727 \times 10^{15}$ \\
\hline \multirow{4}{*}{5} & 0 & 25 & 25 & 25 & 25 \\
\hline & 1 & $-3.50932 \times 10^{12}$ & $-3.53141 \times 10^{12}$ & $-9.95678 \times 10^{11}$ & $-1.00386 \times 10^{12}$ \\
\hline & 2 & $-7.02052 \times 10^{12}$ & $-7.05766 \times 10^{12}$ & $-1.20856 \times 10^{13}$ & $-1.21387 \times 10^{13}$ \\
\hline & 6 & $-2.10687 \times 10^{13}$ & $-2.11533 \times 10^{13}$ & $-6.31281 \times 10^{14}$ & $-6.32312 \times 10^{14}$ \\
\hline
\end{tabular}

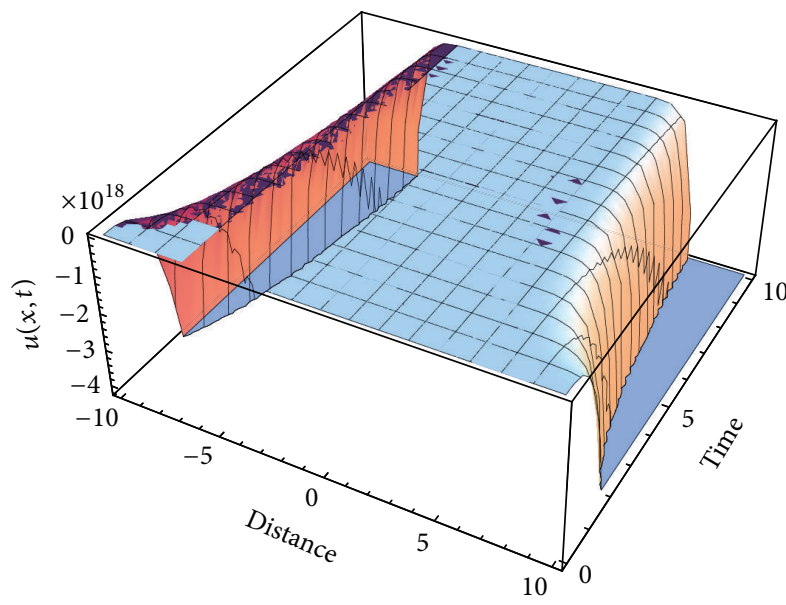

Figure 1: Approximate solution of the time-fractional nonlinear Nagumo equation via the HDM for the value of alpha equal to 0.9 .

Nagumo equation. Figure 1 show, the approximate solution obtained via the HDM for $\alpha=0.9$, Figure 2 shows the approximate solution via the NDVIM. Figure 3 Show the approximate solution obtained via the HDM for $\alpha=0.25$ and Figure 4 shows the approximate solution via the NDVIM. Table 1 shows the comparison of the numerical values of the solution obtained via the HDM and the NDVIM, respectively, for different values of alpha.

Both methods used the idea of iteration; the initial components are obtained as the Taylor series of the exact solution. On one hand, the new development of variational iteration method makes use of the Laplace transform, the Lagrange multiplier, and finally the inverse Laplace transform. On the

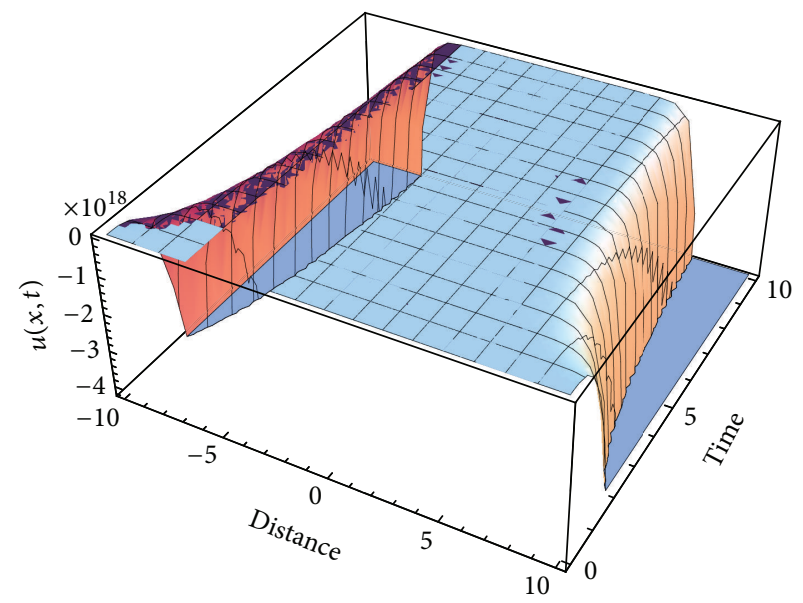

FIgURE 2: Approximate solution of the time-fractional nonlinear Nagumo equation via the NDVIM for the value of alpha equal to 0.9 .

other hand, the HDM uses just a simple integral and the perturbation technique. Both techniques are simple to implement and are very accurate.

\section{Conclusion}

The Nagumo equation is a very complex equation, for which the exact solution does not exist. The Nagumo equation was extended to the concept of fractional order derivative. The resulting equation was further analyzed within the framework of the homotopy decomposition method and the new development of variational iteration method. Both methods use a simple idea of integral transform. The numerical results 


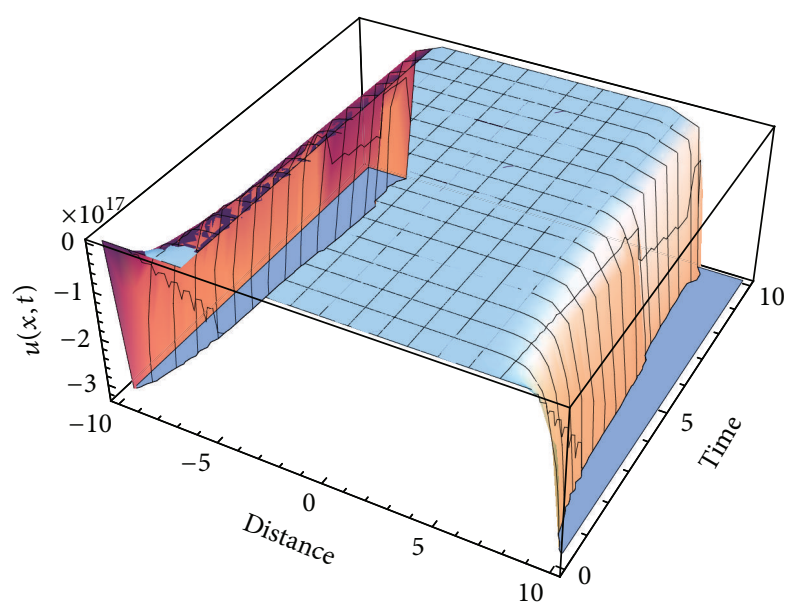

FIGURE 3: Approximate solution of the time-fractional nonlinear Nagumo equation via the HDM for the value of alpha equal to 0.25.

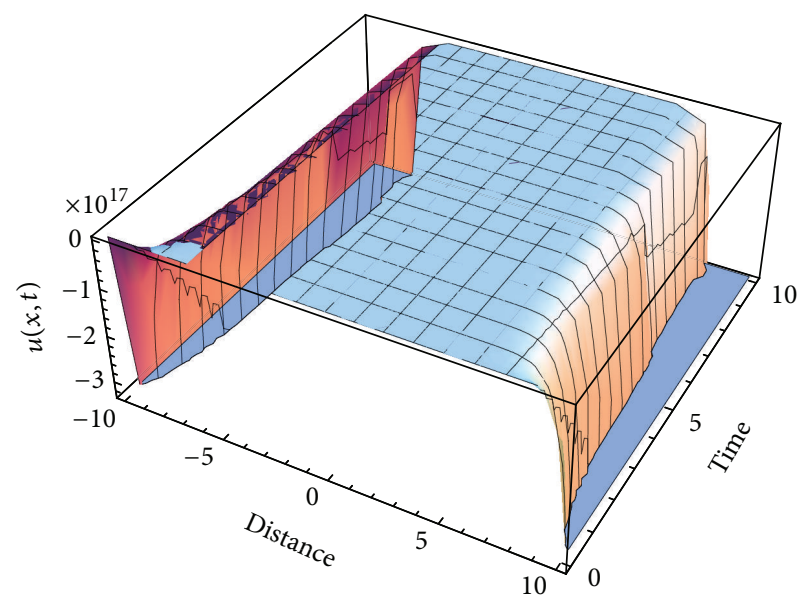

Figure 4: We present in Table 1 the numerical values of the approximate solutions obtained via both methods for different values of alpha.

are presented to test the efficiency and the accuracy of both methods. From their iteration formulas, one can conclude that these two methods are simple to be used and are powerful weapons to handle fractional nonlinear equation type.

\section{Conflict of Interests}

The authors declare that there is no conflict of interests regarding the publication of this paper.

\section{Acknowledgment}

Abdon Atangana would like to thank Claude Leon foundation for their financial support.

\section{References}

[1] D. G . Aronson, "The role of the diffusion in mathematical population biology: skellam revisited," in Mathematics in Biology and Medicine, vol. 57 of Lecture Notes in Biomathematics, Springer, Berlin, Germany, 1985.

[2] J. D. Murray, Mathematical Biology, vol. 19, Springer, Berlin, Germany, 1989.

[3] M. A. Lewis and P. Kareiva, "Allee dynamics and the spread of invading organisms," Theoretical Population Biology, vol. 43, no. 2, pp. 141-158, 1993.

[4] Y. Hosono, "Traveling wave solutions for some density dependent diffusion equations," Japan Journal of Applied Mathematics, vol. 3, no. 1, pp. 163-196, 1986.

[5] P. Grindrod and B. D. Sleeman, "Weak travelling fronts for population models with density dependent dispersion," Mathematical Methods in the Applied Sciences, vol. 9, no. 4, pp. 576586, 1987.

[6] F. Sánchez-Garduño and P. K. Maini, "Travelling wave phenomena in non-linear diffusion degenerate Nagumo equations," Journal of Mathematical Biology, vol. 35, no. 6, pp. 713-728, 1997.

[7] R. Laister, A. T. Peplow, and R. E. Beardmore, "Finite time extinction in nonlinear diffusion equations," Applied Mathematics Letters, vol. 17, no. 5, pp. 561-567, 2004.

[8] M. B. A. Mansour, "Accurate computation of traveling wave solutions of some nonlinear diffusion equations," Wave Motion, vol. 44, no. 3, pp. 222-230, 2006.

[9] J. Naoumo, S. Arimoto, and S. Yoshizawa, "An active pulse transmission line simulating nerve axon," Proceedings of the Institute of Radio Engineers, vol. 50, pp. 2061-2070, 1962.

[10] A. C. Scott, "Neunstor propagation on a tunnel diode loaded transmission line," Proceedings of the IEEE, vol. 51, pp. 240-249, 1963.

[11] J. Nagumo, S. Yoshizawa, and S. Arimoto, "B is table transmission lines," IEEE Transactions on Circuit Theory, vol. 12, pp. 400412, 1965.

[12] J. McKean, "Nagumo's equation," Advances in Mathematics, vol. 4, pp. 209-223, 1970.

[13] S. Hastings, “The existence of periodic solutions to Nagumo's equation," The Quarterly Journal of Mathematics, vol. 25, pp. 369-378, 1974.

[14] C. Zhi-Xiong and G. Ben-Yu, "Analytic solutions of the Nagumo equation," IMA Journal of Applied Mathematics, vol. 48, no. 2, pp. 107-115, 1992.

[15] M. B. A. Mansour, "On the sharp front-type solution of the Nagumo equation with nonlinear diffusion and convection," PRAMANA Journal of Physics, vol. 80, no. 3, pp. 533-538, 2013.

[16] A. Atangana and A. Kilicman, "Analytical solutions of the spacetime fractional derivative of advection dispersion equation," Mathematical Problems in Engineering, vol. 2013, Article ID 853127, 9 pages, 2013.

[17] M. M. Meerschaert and C. Tadjeran, "Finite difference approximations for fractional advection-dispersion flow equations," Journal of Computational and Applied Mathematics, vol. 172, no. 1, pp. 65-77, 2004.

[18] M. Caputo, "Linear models of dissipation whose Q is almost frequency independent-part II," Geophysical Journal International, vol. 13, no. 5, pp. 529-539, 1967.

[19] A. Cloot and J. F. Botha, "A generalised groundwater flow equation using the concept of non-integer order derivatives," Water SA, vol. 32, no. 1, pp. 1-7, 2006. 
[20] D. A. Benson, S. W. Wheatcraft, and M. M. Meerschaert, "Application of a fractional advection-dispersion equation," Water Resources Research, vol. 36, no. 6, pp. 1403-1412, 2000.

[21] G. Wu and D. Baleanu, "Variational iteration method for the Burgers' flow with fractional derivatives-new Lagrange multipliers," Applied Mathematical Modelling, vol. 37, no. 9, pp. 61836190, 2013.

[22] M. Matinfar and M. Ghanbari, "The application of the modified variational iteration method on the generalized Fisher's equation," Journal of Applied Mathematics and Computing, vol. 31, no. 1-2, pp. 165-175, 2009.

[23] Y. Tan and S. Abbasbandy, "Homotopy analysis method for quadratic Riccati differential equation," Communications in Nonlinear Science and Numerical Simulation, vol. 13, no. 3, pp. 539-546, 2008.

[24] J. S. Duan, R. Rach, D. Baleanu, and A. M. Wazwaz, "A review of the Adomian decomposition method and its applications to fractional differential equations," Communications in Fractional Calculus, vol. 3, no. 2, pp. 73-99, 2012.

[25] M. Javidi and M. A. Raji, "Combinaison of Laplace transform and homotopy perturbation method to solve the parabolic partial differential equations," Communications in Fractional Calculus, vol. 3, pp. 10-19, 2012.

[26] K. S. Miller and B. Ross, An Introduction to the Fractional Calculus and Fractional Differential Equations, A Wiley-Interscience Publication, John Wiley \& Sons, New York, NY, USA, 1993.

[27] I. Podlubny, "Geometric and physical interpretation of fractional integration and fractional differentiation," Fractional Calculus \& Applied Analysis, vol. 5, no. 4, pp. 367-386, 2002.

[28] A. Anatoly, J. Juan, and M. S. Hari, Theory and Application of Fractional Differential Equations, Elsevier, Amsterdam, The Netherlands, 2006.

[29] D. Baleanu, K. Diethelm, E. Scalas, and J. J. Trujillo, Fractional Calculus: Models and Numerical Methods, Complexity, Nonlinearity and Chaos, World Scientific, 2012.

[30] A. A. Kilbas, H. M. Srivastava, and J. J. Trujillo, Theory and Applications of Fractional Differential Equations, vol. 204 of NorthHolland Mathematics Studies, Elsevier Science B.V., Amsterdam, The Netherland, 2006.

[31] M. Madani, M. Fathizadeh, Y. Khan, and A. Yildirim, "On the coupling of the homotopy perturbation method and Laplace transformation," Mathematical and Computer Modelling, vol. 53, no. 9-10, pp. 1937-1945, 2011.

[32] Y. Khan and H. Latifizadeh, "Application of new optimal homotopy perturbation and Adomian decomposition methods to the MHD non-Newtonian fluid flow over a stretching sheet," International Journal of Numerical Methods for Heat \& Fluid Flow, vol. 24, no. 1, pp. 124-136, 2014.

[33] D. Baleanu, O. G. Mustafa, and R. P. Agarwal, "On the solution set for a class of sequential fractional differential equations," Journal of Physics A: Mathematical and Theoretical, vol. 43, no. 38, Article ID 385209, 7 pages, 2010.

[34] D. Baleanu, H. Mohammadi, and S. Rezapour, "Some existence results on nonlinear fractional differential equations," Philosophical Transactions of the Royal Society of London A, vol. 371, no. 1990, Article ID 20120144, 2013.

[35] J.-F. Cheng and Y.-M. Chu, "Fractional difference equations with real variable," Abstract and Applied Analysis, vol. 2012, Article ID 918529, 24 pages, 2012.

[36] F. Jarad, T. Abdeljawad, D. Baleanu, and K. Biçen, "On the stability of some discrete fractional nonautonomous systems,"
Abstract and Applied Analysis, vol. 2012, Article ID 476581, 9 pages, 2012.

[37] T. Abdeljawad and D. Baleanu, "Caputo $q$-fractional initial value problems and a $q$-analogue Mittag-Leffler function," Communications in Nonlinear Science and Numerical Simulation, vol. 16, no. 12, pp. 4682-4688, 2011.

[38] K. Diethelm, E. Scalas, and D. Baleanu, Fractional Calculus Series on Complexity of Series on Complexity, Nonlinearity and Chaos, vol. 3, World Scientific Publishing, 2012.

[39] A. Atangana and J. F. Botha, "Analytical solution of groundwater flow equation via homotopy decomposition method," Journal of Earth Science \& Climatic Change, vol. 3, no. 115, p. 2157, 2012.

[40] A. Atangana and A. Secer, "The time-fractional coupled-Korteweg-de-Vries equations," Abstract and Applied Analysis, vol. 2013, Article ID 947986, 8 pages, 2013.

[41] A. Atangana and E. Alabaraoye, "Solving system of fractional partial differential equations arisen in the model of HIV infection of $\mathrm{CD} 4^{+}$cells and attractor one-dimensional KellerSegel equation," Advances in Difference Equations, vol. 2013, article 94, 14 pages, 2013.

[42] Y. Khan and Q. Wu, "Homotopy perturbation transform method for nonlinear equations using He's polynomials," Computers \& Mathematics with Applications, vol. 61, no. 8, pp. 19631967, 2011.

[43] G. C. Wu, "New trends in the variational iteration method," Communications in Fractional Calculus, vol. 2, no. 2, pp. 59-75, 2011.

[44] G. Wu and D. Baleanu, "Variational iteration method for fractional calculus-a universal approach by Laplace transform," Advances in Difference Equations, vol. 2013, article 18, 2013. 


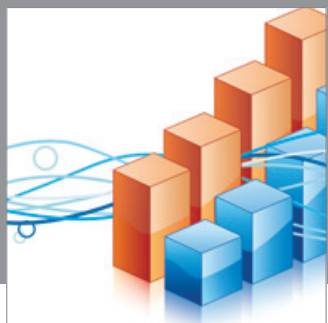

Advances in

Operations Research

mansans

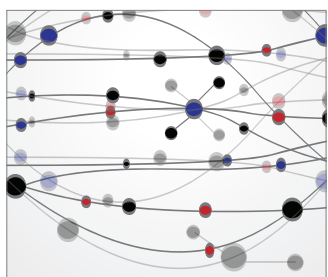

The Scientific World Journal
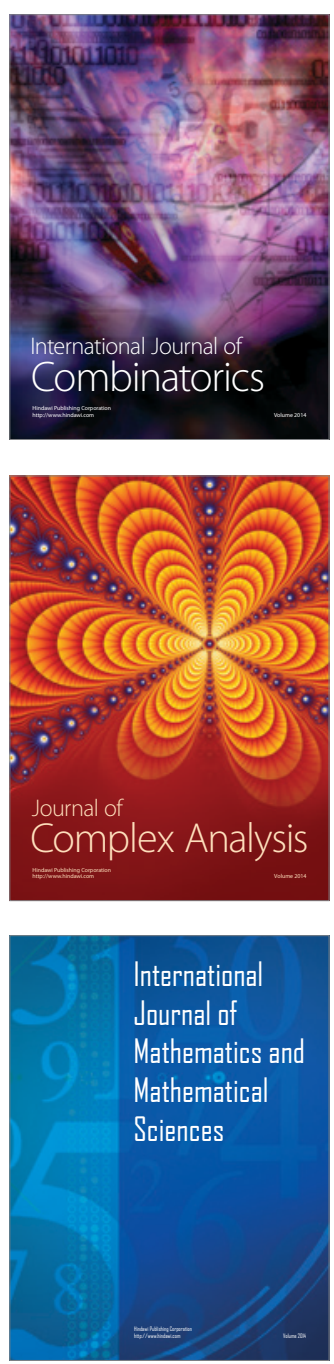
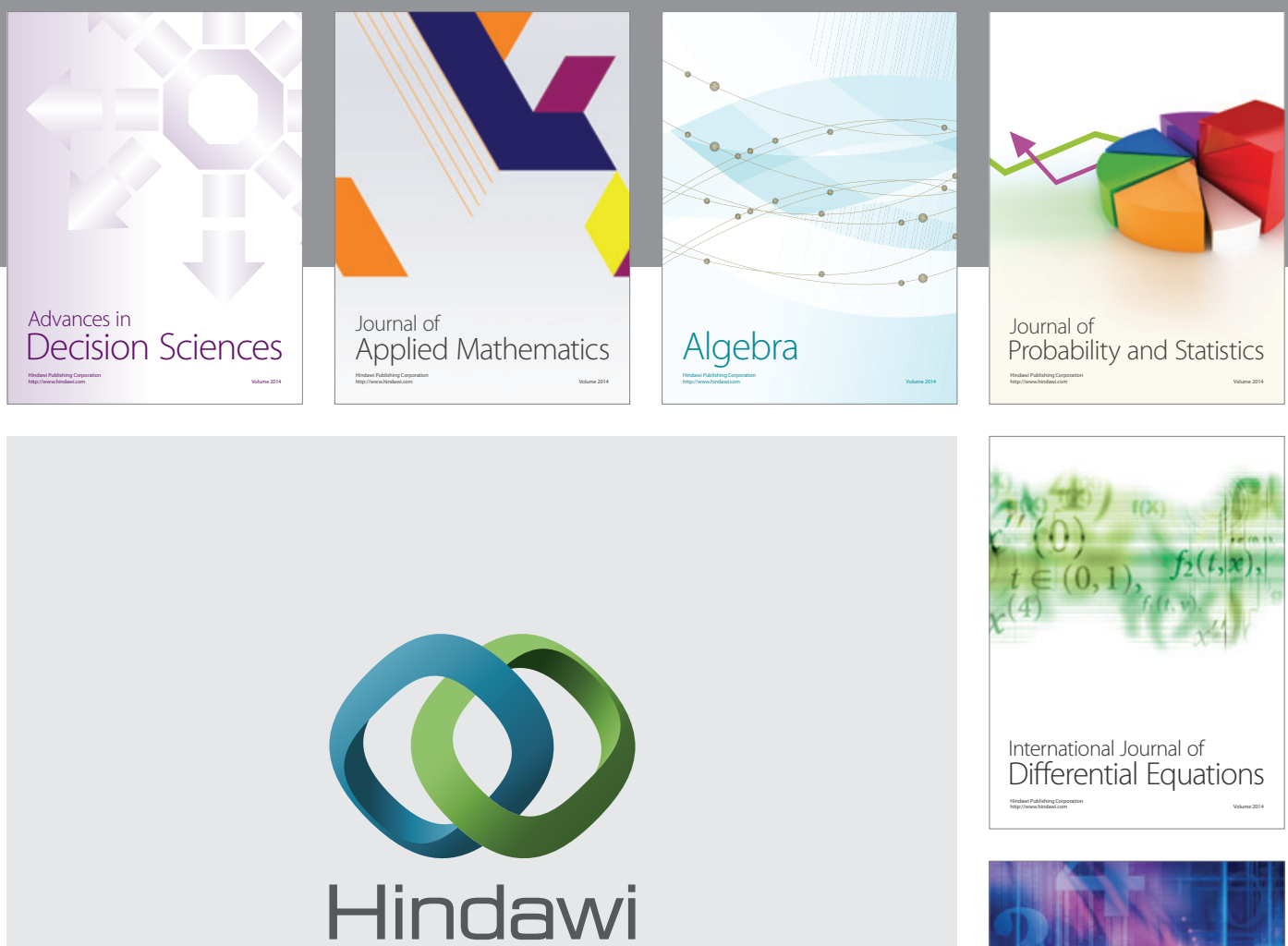

Submit your manuscripts at http://www.hindawi.com
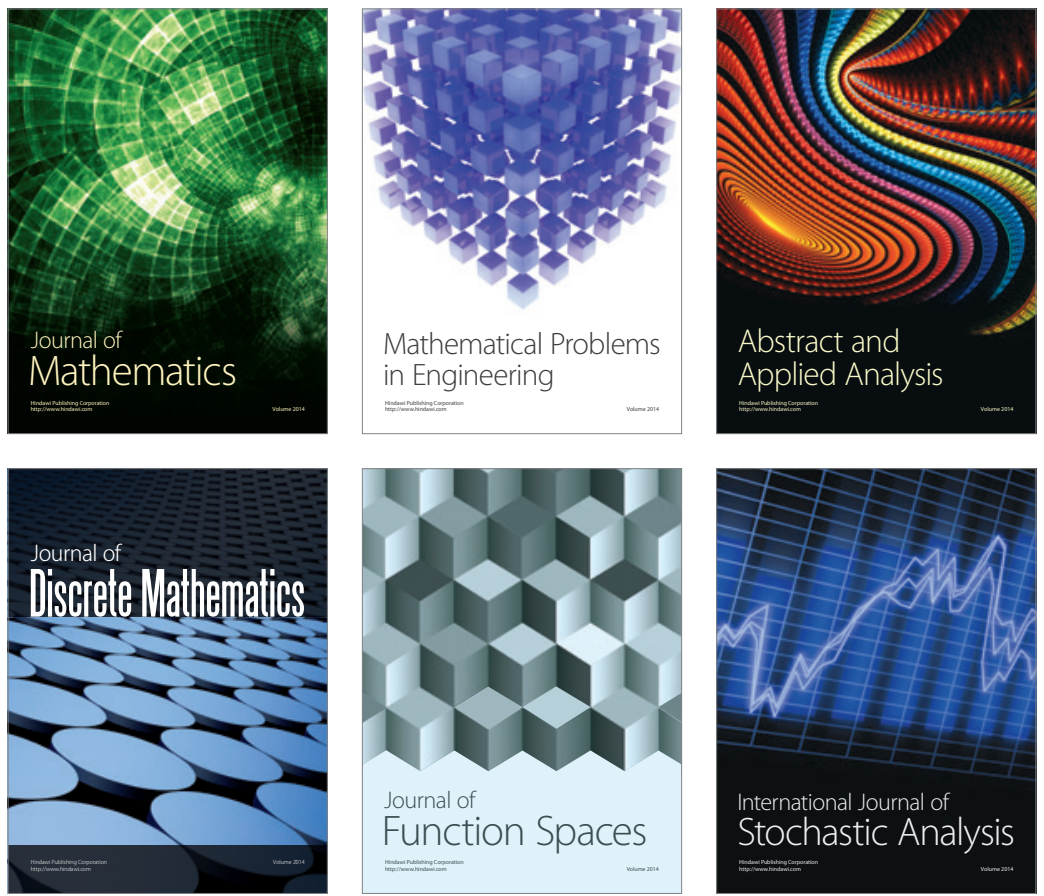

Journal of

Function Spaces

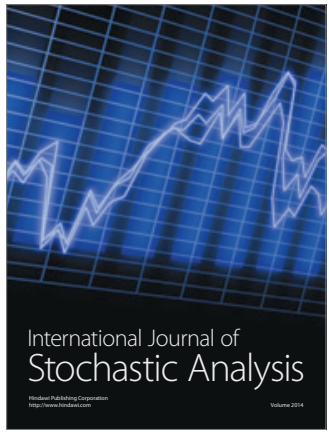

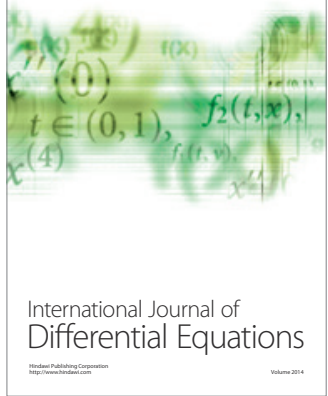
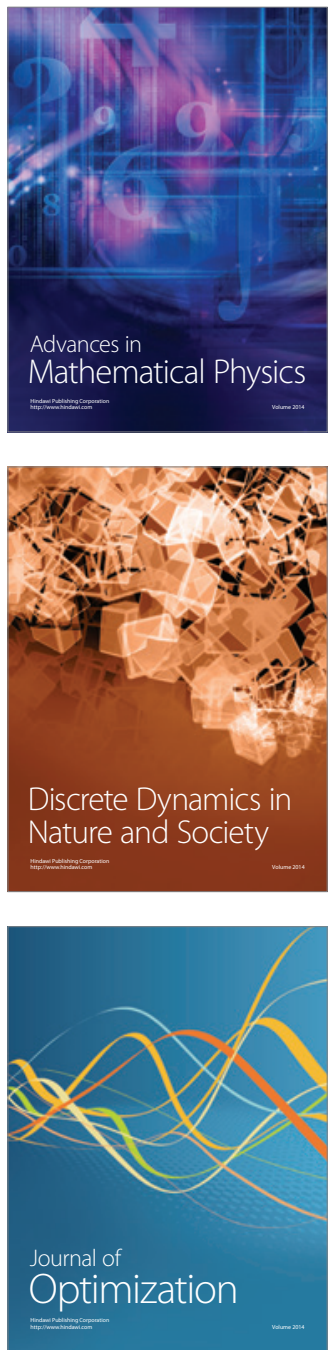